\title{
Twenty years of the Critical Psychiatry Network
}

\author{
Duncan B. Double
}

The Critical Psychiatry Network (CPN) was formed in 1999. This editorial attempts to define critical psychiatry and notes some key contributions from members of the CPN. The implications of critical psychiatry and some differences within the critical psychiatry movement are discussed.

\section{Declaration of interest}

D.B.D is founding member of the Critical Psychiatry Network.

\section{Keywords}

History of psychiatry; philosophy; aetiology.

\section{Copyright and usage}

(c) The Royal College of Psychiatrists 2019.
Duncan Double is a part-time Consultant Psychiatrist at Norfolk and Suffolk National Health Service Foundation Trust and is currently doing a part-time PhD at Cambridge University on 'The foundations of critical psychiatry'. He blogs on critical psychiatry at www.criticalpsychiatry.blogspot.com.
The Critical Psychiatry Network (CPN) first met in January 1999 because of concern about the potential for increasing coercion in the context of reform of the Mental Health Act 1983, which eventually led to the 2007 amendments. Initially, it was called the Bradford Group after the city in which the first meeting took place. By the sixth meeting in October 1999, the name 'Critical Psychiatry Network' had been adopted, reflecting that the group had become more explicit about its wider critical psychiatry concerns. CPN has continued to meet on a roughly biannual basis.

\section{Definition of critical psychiatry}

Critical psychiatry may be difficult to define precisely. It has never hidden its association with so-called anti-psychiatry, which is particularly associated with the psychiatrists R. D. Laing and Thomas Szasz (although both rejected this designation). In some ways, the term anti-psychiatry has generally been used within mainstream psychiatry in response to criticism which it does not accept.

Mainstream psychiatry is predicated on the notion that mental illness is brain disease. The essential position of critical psychiatry is that functional mental illness should not be reduced to brain disease. This statement should not be misunderstood; there is, of course, no controversy about the fact that brains enable minds. However, there is a need to move beyond a mental health system based on the 'disease model'. ${ }^{1}$ Functional mental illness is a personal experience that does not have an underlying brain pathology. ${ }^{2}$ No definite biomarkers have been linked to functional mental illness and inconsistencies and confounders plague research studies.

The degree of dogmatism among psychiatrists about the disease model varies. Many insist their position is eclectic and biopsychosocial rather than narrowly biomedical. This commonly held perspective was articulated by Anthony Clare ${ }^{3}$ as the outcome of the anti-psychiatry debate. He eschewed a well-defined basis for practice to avoid the worst excesses of reductionism and the objectification of patients, which was of such concern to anti-psychiatry. Manschrek and Kleinman ${ }^{4}$ called this kind of position 'semi-critical', because it fails to appreciate fully the limitations of the biomedical model and thus does not push the necessary critique far enough. By contrast, they called biomedical dogmatism the 'hubris position' and saw it as 'dangerous'.

As an illustration, George Engel ${ }^{5}$ - in proposing his biopsychosocial model - was responding to an article by Ludwig ${ }^{6}$ entitled 'The psychiatrist as physician'. Ludwig was concerned about anti-psychiatric critiques. His response was to accept the vulnerability of psychiatry to such charges and his solution was to retreat to the medical model. As far as Ludwig was concerned, psychiatry should deal with medical illness, including neuropsychiatric and medico-psychiatric disorders, rather than non-psychiatric problems, which are more appropriately handled by nonmedical professionals. Ludwig was clear that psychiatry's viability was dependent on an understanding of mental illness as due to known, suggestive or presumed brain dysfunction. Engel, by contrast, was not happy with Ludwig's attempt to salvage psychiatry in this way in the context of anti-psychiatry. As far as he was concerned, all medicine, not just psychiatry, is in crisis. He believed doctors had become insensitive to the personal problems of patients and were preoccupied with procedures. In short, medicine is too disease oriented rather than patient oriented. For Engel, the biopsychosocial model has the real advantage of taking account of cultural, social and psychological considerations as well as biological factors. Furthermore, it avoids the polarisation between biomedical reductionists (among whom Engel would have included Ludwig) and exclusionists (like Thomas Szasz) who deny mental illness. Critical psychiatry applies the patient-centred method and adopts the biopsychosocial model, although not in the eclectic, atheoretical way in which it tends to be used in modern psychiatry.

\section{Strands of critical psychiatry}

Modern critical psychiatry has several strands. For reasons of space, this editorial has to be selective about the contributions from key members of the CPN. For example, Sami Timimi ${ }^{7}$ describes feeling confused by what he experienced as the indoctrination in his psychiatric training. Believing that mental illness is a brain disease is more like a faith that doctors are obliged to believe rather than a scientific position. As a child and adolescent psychiatrist, Sami expresses concern about how this field has become increasingly biologized over recent years.

Perhaps the most well-known version of critical psychiatry is postpsychiatry, ${ }^{8}$ which started as a series of articles in the OpenMind magazine. Tying critical psychiatry to postmodernism, 
however, is controversial and Pat Bracken and Phil Thomas do not see postmodernism as a rejection of other critical positions.

Although anti-psychiatry had little to do with issues of race and culture, this element has been central for critical psychiatry, as represented for example in the work of Suman Fernando. ${ }^{9}$ Lowand middle-income countries need to develop locally relevant approaches rather than follow the biomedical methods of highincome countries, thereby acknowledging how culturally embedded mental health problems are in the social, economic and political conditions of countries.

Another difference from anti-psychiatry is that critical psychiatry is prepared to engage with the evidence about psychiatric treatment. It takes a sceptical approach, emphasising the bias in the literature created by methodological problems with clinical trials, such as unblinding. For example, placebo amplification is seen as a valid explanation of the data in antidepressant trials. Critical psychiatry is not so much making a case for 'the myth of mental illness', as was Thomas Szasz, but for 'the myth of the chemical cure' (as in the title of the book by Joanna Moncrieff ${ }^{10}$ ).

\section{Implications of critical psychiatry}

Critical psychiatry has implications for both diagnosis and treatment. As far as diagnosis is concerned, critical psychiatrists are not so interested in arriving at a single word diagnosis. If mental illness is not a brain disease, there is no need to specify an underlying brain problem. Instead the focus is on understanding the person and why they have presented with the problems they have in the context of their life situation.

In relation to treatment, critical psychiatrists operate within the framework of the Mental Health Act, taking a rights-based approach. They are not just psychotherapists who see people on a voluntary basis, although they do facilitate people seeking psychotherapy if they wish. They try to minimise the use of coercion and have been against the introduction of community treatment orders. They are not against psychotropic medication, which they prescribe within the uncertainty of the available evidence. They are very aware of the risk of discontinuation problems from medication. The emphasis in treatment is on helping people improve their social situation and to be as independent as they want to be.

Therefore, too much can be made of the difference between the practices of critical and mainstream psychiatry. However, critical psychiatry's challenge to reductionism and positivism, including mechanistic psychological approaches, does create a framework which focuses on the person and has ethical, therapeutic and political implications for clinical practice. It also has consequences for psychiatric research, which has become too focused on speculative neurobiological notions.

\section{Differences within the critical psychiatry movement}

Critical psychiatry is a broad school of thought and is now used as a general term for alternatives to biomedical psychiatry (e.g. by the 'Mad in America' website, which includes the perspectives of psychiatric patients and survivors; www.madinamerica.com). At least three main areas of disagreement within critical psychiatry can be discerned, although these issues may not be totally distinct:

(a) Should psychiatry be seen as a medical discipline? There is agreement that psychiatry is different from medicine, but the disagreement arises from how much is made of that difference. Some people want to go as far as saying that psychiatry should be nonmedical; others emphasise that medicine covers both physical and mental aspects. The reality is that many people do complain of physical symptoms which have psychosocial origins and psychosomatic aspects of medicine have to be acknowledged.

(b) Should the Mental Health Act be abolished? The emphasis on the rights of people with mental health problems leads some to argue for abolishing all forced treatment and others to accept that detention can be justified by the loss of mental capacity in mental illness. Psychiatric abuse is of course unjustifiable and critical psychiatry attempts to minimise the use of coercion.

(c) Is it suitable to see mental disorder as illness and disease? Since mental disorder is not a brain disease, some questions arise: should it be seen as illness, and is psychiatric diagnosis valid? Some people conclude that mental disorder is not illness and alternatives are required to psychiatric diagnosis, while others accept that psychological dysfunction can be understood as illness and that diagnostic concepts should be understood for what they are. Diagnoses should not be reified and seen as 'things'. Instead they are merely idealised, hypothetical constructs and if they have any value they should be understood as such.

\section{Conclusion}

Even after 20 years, the CPN seems marginal to mainstream psychiatry and seeks more recognition of the validity of its position. The CPN does, however, accept that the wish to find a physicalist basis for mental illness will never go away. Further debate on taking psychiatry beyond the current dominant paradigm is required.

Duncan B. Double, MB, BChir, MRCPsych, Consultant Psychiatrist, Adult Psychiatry, Norfolk and Suffolk National Health Service Foundation Trust, UK

Correspondence: Duncan B. Double, Victoria House, 28 Alexandra Road, Lowestoft, Suffolk, NR32 1PL, UK. Email: dbdouble@dbdouble.co.uk

First received 13 Jul 2018, accepted 12 Aug 2018

\section{References}

1 British Psychological Society Division of Clinical Psychology. Classification of Behaviour and Experience in Relation to Functional Psychiatric Diagnosis: Time for a Paradigm Shift. British Psychological Society, 2013.

2 Double DB (ed). Critical Psychiatry: The Limits of Madness. Palgrave Macmillan, 2006.

3 Clare A. Psychiatry in Dissent: Controversial Issues in Thought and Practice. Tavistock Publications, 1976

4 Manschrek TC, Kleinman A (eds). Renewal in Psychiatry: A Critical Rational Perspective. Wiley, 1977.

5 Engel G. The need for a new medical model: a challenge for biomedicine. Science 1977; 196: 129-36.

6 Ludwig A. The psychiatrist as physician. JAMA 1975; 234: 603-4.

7 Timimi S. Pathological Child Psychiatry and the Medicalization of Childhood Brunner-Routledge, 2002.

8 Bracken P, Thomas P. Postpsychiatry: Mental Health and Postmodernism. Oxford University Press, 2005.

9 Fernando S. Mental Health Worldwide: Culture, Globalisation and Development. Palgrave Macmillan, 2014.

10 Moncrieff J. The Myth of the Chemical Cure: A Critique of Psychiatric Drug Treatment. Palgrave Macmillan, 2008. 